\title{
THE USE OF CAST-BRACING AS TREATMENT FOR FRACTURES OF THE TIBIAL PLATEAU
}

\author{
THOMAS SCOTLAND. DOUGLAS WARDLAW \\ From the Department of Orthopaedic Surgery, Aberdeen Royal Infirmary
}

\begin{abstract}
A series of 29 patients with fractures of the tibial plateau were treated by means of a cast-brace. There were seven wedge fractures of the lateral tibial condyle, two bicondylar fractures and 20 compression fractures involving the lateral tibial plateau.

The seven patients with wedge fractures were treated by skin or skeletal traction, followed as soon as possible by flexion exercises for the knee. Cast-braces were applied to all fractures as soon as possible after injury, and the patients allowed to bear weight freely. Early restoration of function of the injured limb was thereby achieved.

The results of our study over a period of two and a half years indicate that cast-bracing is a very satisfactory method of treating fractures of the tibial plateau. All the fractures united, the movements and control of the knee were excellent, and valgus or varus deformity was unchanged before application and after removal of the brace.
\end{abstract}

There are conflicting views concerning the best treatment for patients with fractures of the tibial plateau. Many surgeons claim that anatomical reduction and stable internal fixation of the fragments is the treatment of choice (Palmer 1939; Rombold 1960). However, this is not always possible particularly in comminuted compression fractures. On the other hand, several long-term studies have demonstrated that conservative treatment is a satisfactory alternative, despite imperfect radiological appearances of the fracture (Slee 1955: Turner 1959; Hohl 1967). Apley's method of skeletal traction and early flexion of the knee (Apley 1956) has gained wide acceptance, although prolonged bed-rest followed by protected weightbearing are two disadvantages of this form of treatment.

Cast-bracing is applicable to all types of fractures of the tibial plateau regardless of whether these fractures are treated primarily by operative means or conservatively by traction. It removes the two disadvantages of Apley's treatment regime by allowing early weightbearing and early discharge from hospital-an important consideration in days of escalating costs and increasing demands for the too few orthopaedic beds. Early weight-bearing means early restoration of function to the injured limb, not only to the joint itself, but to the surrounding muscles and tendons.

Classification of fractures of the tibial plateau depends on an understanding of the underlying mechanism of injury. The sharp anterior part of the lateral femoral condyle may be driven into the underlying tibial plateau like a chisel, shearing off a single large fragment (Rasmussen 1973). This is a "split" or "wedge" fracture (Fig. 1). Depending on the magnitude of force, it may be undisplaced, or greatly displaced and unstable; and depending on the direction of force, one or both tibial condyles may be broken off. Usually, the lateral plateau is involved first and therefore most of the damage is on the lateral side of the joint.

If a larger surface area of the lateral femoral condyle is driven down, there may be crushing of the tibial plateau over a much wider area, resulting in a compression fracture (Fig. 2). The degree of crushing depends primarily on the resistance of subchondral

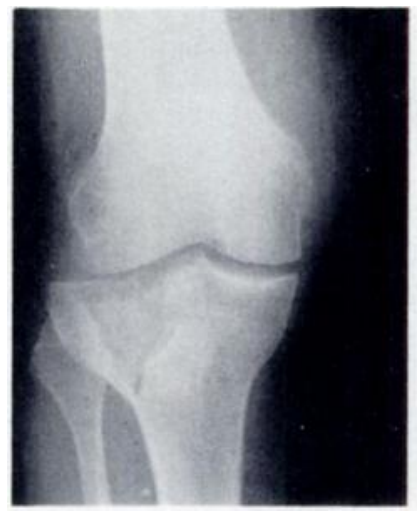

Fig. 1

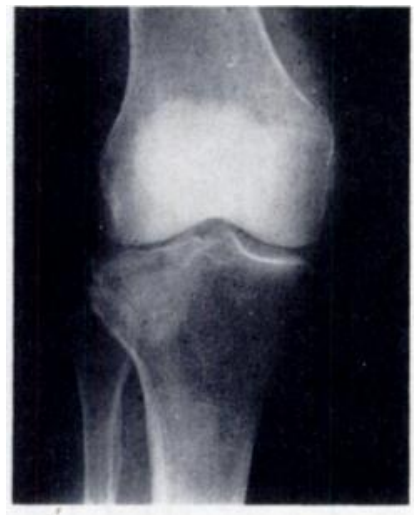

Fig. 2
Figure 1-Radiograph showing the wedge fracture classified by Rasmussen (1973). Figure $2-$ Radiograph showing a compression

T. R. Scotland, FRCS Ed, Senior Registrar

D. Wardlaw, FRCS Ed. Consultant Orthopaedic Surgeon

Requests for reprints should be sent to Mr D. Wardlaw.

- 1981 British Editorial Society of Bone and Joint Surgery 0301-620X/81/4100-0575 $\$ 2.00$ 
bone, which in turn is closely related to the age of the patient. It depends also on the amount of flexion of the knee at the time of injury and the magnitude of the axial force producing the fracture. Many fractures are a combination of wedge and compression injuries, involving a certain amount of central crushing with a wedge of variable size laterally.

\section{MATERIAL AND METHODS}

Twenty-nine patients were treated by means of a cast-brace over a period of two and a half years from October 1977 to March 1980. Their ages ranged from 16 to 82 years with a distribution as shown in Figure 3. There were seven patients with displaced wedge fractures of the lateral tibial condyle, two bicondylar fractures and 20 compression fractures of the lateral plateau.

The seven patients with displaced wedge fractures were, with one

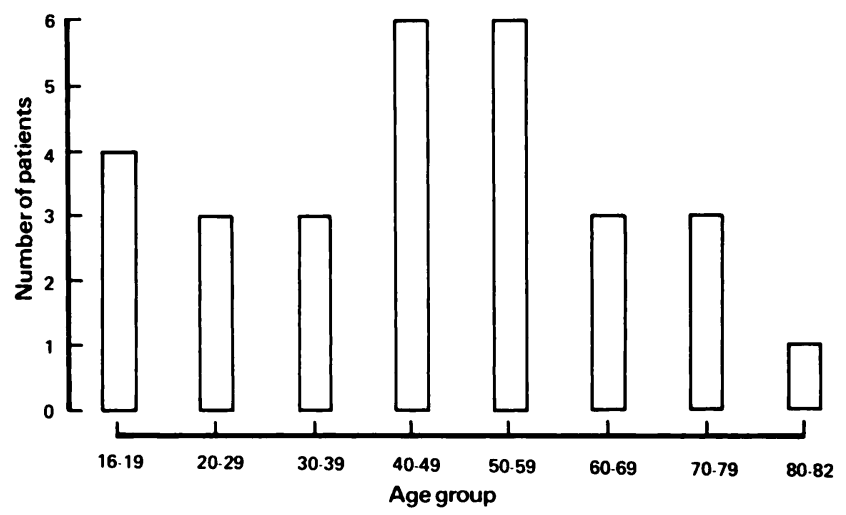

Fig. 3

Histogram showing the distribution of age in the group of 29 patients.

exception, in the age groups under 49 years. The trauma sustained by this grcup was more severe than in those patients with compression fractures. All of these seven patients were treated primarily by internal fixation using one, or where possible two, cancellous-bone screws to restore stability: all had serious valgus instability before operation. The operation was simple and inflicted minimal damage on an already traumatised knee. After operation the patients were treated in tube plasters for 10 to 16 days and cast-braces were applied after removal of the stitches. There was no problem with wound healing in any of this group.

Twenty of the remaining patients, had compression fractures and two had bicondylar fractures. They were treated, where possible, by

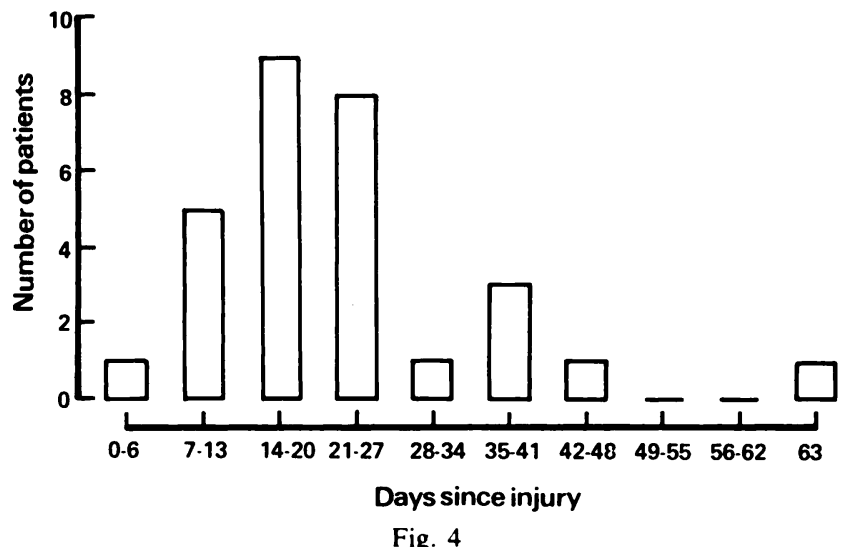

skin traction for a few days until swelling of the joint had disappeared and when flexion of the knee to 60 degrees was possible without pain. Cast-braces were then applied. Modification of this regime was necessary in patients with associated injuries to the legs (Table I).

All patients, whether treated by operation or conservatively, had a cast-brace applied as soon as possible after injury. The time interval

Table I. Associated injuries in the legs of 9 patients with fractures of the tibial plateau (Total 12 fractures in 9 patients)

\begin{tabular}{|l|c|c|}
\hline & Same leg & Opposite leg \\
\hline Intertrochanteric fracture & 1 & - \\
Midshaft femur & 3 & 1 \\
Lowermost third of femur & 1 & - \\
Patella & - & 1 \\
Midshaft tibia and fibula & 1 & 3 \\
Distal tibia and fibula & - & 1 \\
\hline
\end{tabular}

between injury and application of the brace was noted. At the time of application the range of knee movements, extensor lag and valgus or varus deformity of the knee were measured. Patients were allowed to bear weight as soon as the cast was dry, and in most cases were allowed home within a few days.

Several weeks later, after the fracture had healed and the brace had been removed, the above measurements were repeated and the time spent in a brace recorded. At the end of treatment, a functional grading system based on the classification by Rasmussen (1973) was employed to evaluate the results.

\section{RESULTS}

The time of application of the brace varied from 6 to 63 days after injury; delay was invariably due to associated injury. If one splits the patients into two groups, those with tibial plateau fractures only and those with associated injury, it can be seen that in uncomplicated fractures of the tibial plateau all but one patient had the brace applied within three weeks of injury. Associated fractures of the femoral shaft delayed application, although fractures of the tibia did not lengthen the period of immobilisation (Figs 4 and 5).

The time spent in a cast-brace varied from 3 to 12

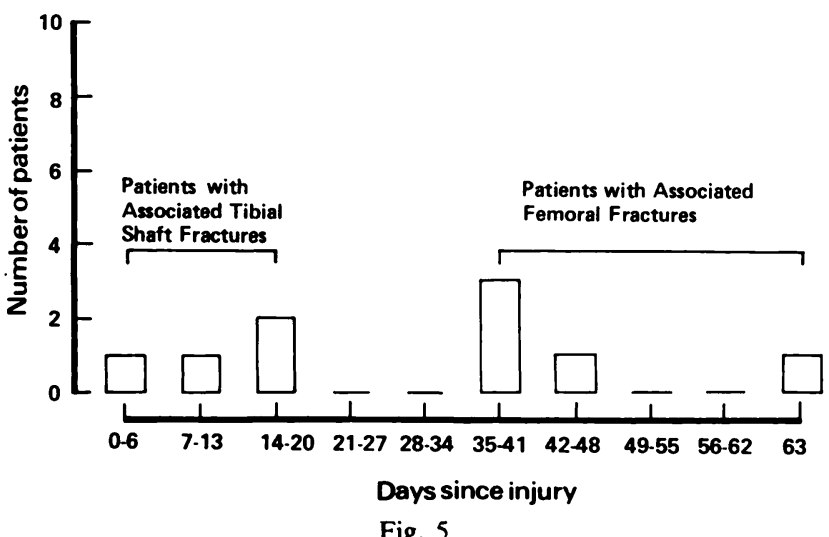

Fig. 5

Figure 4-Time of application of cast-brace after injury in 29 patients with fractures of the tibial plateau. Figure 5-Time of application of the cast-brace in nine patients with fractures of the tibial plateau associated with other injuries of the leg. 
weeks (Fig. 6). Two patients were lost to the series at this stage; one left the area and was lost to follow-up and the other died of a gastric carcinoma. Patients with associated fractures of the femoral shaft remained in their brace for a longer time.

In all but one patient the valgus or varus deformity of the knee remained the same before and after treatment with a brace. In the one patient with a

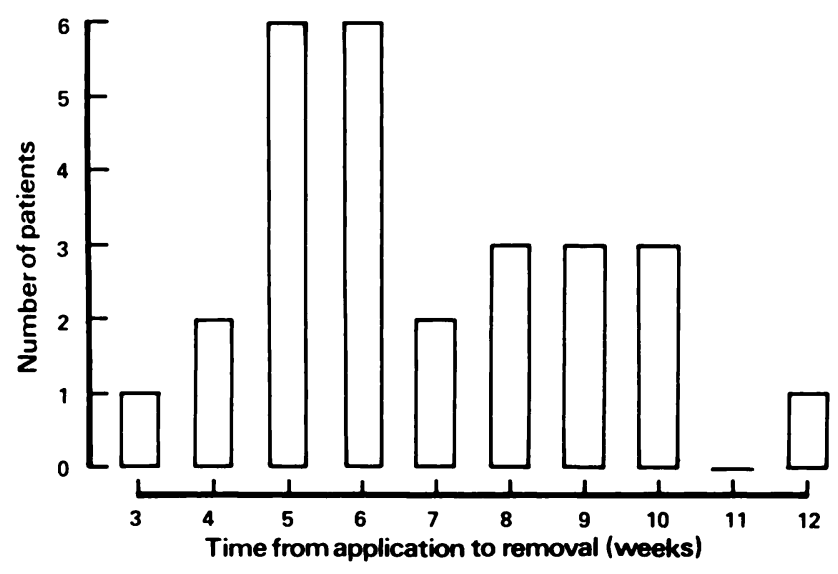

Fig. 6

Histogram showing the time spent in a cast-brace for 27 patients.

combined compression fracture with a small lateral wedge the initial deformity was zero degrees. He was offered internal fixation but declined. The wedge displaced and he ended up with a valgus deformity of five degrees. This patient was also psychotic and did not always cooperate fully. These results emphasise the point that we do not necessarily advocate cast-bracing as an alternative to operation. Where unstable fractures exist, they should first be stabilised by internal fixation.

One of the problems of assessing results in fractures of the tibial plateau is that different workers measure different parameters. Rasmussen (1973) introduced a functional grading system which has gained wide acceptance. Pain, the capacity to walk, extensor lag, the total range of movements, and stability of the knee are assessed using a points system. Results are classified as acceptable or unacceptable depending on the total number of points awarded.

Using Rasmussen's classification 10 patients had an excellent result, 16 patients a good result and only one patient, in whom the pain was incapacitating, had an unacceptable result. Eight patients experienced occasional aches in bad weather and the other 18 had no pain.
The capacity to walk was normal in relation to age in 16 of our patients; good in 10 patients who all managed quite long walks out of doors; and poor in one patient whose activity was restricted by pain.

Seventeen patients had full extension, while the other 10 had a five degree extensor lag. All but one of the patients had an excellent or good range of movement in excess of 90 degrees. The one exception was a patient who had a stellate fracture of her patella and a compound supracondylar fracture of the femur on the same side. She only achieved 60 degrees of flexion.

Residual instability was never severe enough to spoil the end result in any of our patients.

\section{DISCUSSION}

The aim of treating fractures of the tibial plateau is to produce a painless stable knee with a good range of movements supported by strong muscles. Cast-bracing of the injured limb allows early mobilisation, whereas weight-bearing encourages recovery of normal muscle action around the knee and promotes the flow of blood through the tissues. The injured limb is therefore restored to its former physiological state a short time after the fracture occurs.

Our results compare very favourably with other series. In Rasmussen's very large series of 260 patients, 44 per cent had operative treatment, and 87 per cent had acceptable results. Brown and Sprague (1976) treated 29 patients by cast-bracing and their results, evaluated using Rasmussen's system, were comparable to ours. DuParc and Ficat (1960) operated on 54 per cent of 144 patients. The results were acceptable in 62 per cent of the patients who had not been operated on, and in 84 per cent of those who had.

The results in this series indicate that cast-bracing controls what was considered to be an acceptable valgus or varus deformity. It should be emphasised that full weight-bearing in a cast-brace can be allowed without fear of increasing valgus displacement.

The principle of early mobilisation by quadriceps exercises and flexion exercises for the knee followed by protected weight-bearing revolutionised the treatment of fractures of the tibial plateau (Apley 1956). However, it suffered from two major disadvantages, prolonged bed-rest and protected weight-bearing. Cast-bracing eliminates these two disadvantages whilst retaining the advantages of Apley's form of treatment and provides a step forward in the treatment of fractures of the tibial plateau.

We wish to acknowledge the help and support of the consultant orthopaedic surgeons, Grampian Health Board, without whose assistance this paper would not have been possible. 
REFERENCES

Apley AG. Fractures of lateral tibial condyle treated by skeletal traction and early mobilisation: a review of sixty cases with special reference to the long-term results. J Bone Joint Surg [Br] 1956;38-B:699-708.

Brown GA, Sprague BL. Cast brace treatment of plateau and bicondylar fractures of the proximal tibia. Clin Orthop 1976;119:184-93.

DuParc J, Ficat P. Fractures articulaires de l'extrémité supérieure du tibia. Rev Chir Orthop 1960:46:339-486.

Hohl M. Tibial condylar fractures. J Bone Joint Surg [Am] 1967;49-A:1455-67.

Palmer I. Compression fractures of the lateral tibial condyle and their treatment. J Bone Joint Surg 1939:21:674-80.

Rasmussen PS. Tibial condylar fractures: impairment of knee joint stability as an indication for surgical treatment. J Bone Joint Surg [Am] 1973:55-A:1331-51.

Rombold C. Depressed fractures of the tibial plateau. J Bone Joint Surg [Am] 1960;42-A:783-97.

Slee GC. Fractures of the tibial condyles. J Bone Joint Surg [Br] 1955;37-B:427-37.

Turner VC. Fractures of the tibial plateaus. JAMA 1959;169:923-6. 